\title{
KALwEN+: Practical Key Management Schemes for Gossip-Based Wireless Medical Sensor Networks
}

\author{
Zheng Gong ${ }^{1}$, Qiang Tang ${ }^{1}$, Yee Wei Law ${ }^{2}$ and Hongyang Chen $^{3}$ \\ 1 Faculty of EWI, University of Twente, The Netherlands \\ \{z.gong, q.tang\}@utwente.nl \\ 2 Department of EEE, The University of Melbourne, Australia \\ yee.wei.law@gmail.com \\ 3 Institute of Industrial Science, The University of Tokyo, Japan \\ hongyang@mcl.iis.u-tokyo.ac.jp
}

\begin{abstract}
The constrained resources of sensors restrict the design of a key management scheme for wireless sensor networks (WSNs). In this work, we first formalize the security model of ALwEN, which is a gossipbased wireless medical sensor network (WMSN) for ambient assisted living. Our security model considers the node capture, the gossip-based network and the revocation problems, which should be valuable for ALwENlike applications. Based on Shamir's secret sharing technique, we then propose two key management schemes for ALwEN, namely the KALwEN+ schemes, which are proven with the security properties defined in the security model. The KALwEN+ schemes not only fit ALwEN, but also can be tailored to other scalable wireless sensor networks based on gossiping.
\end{abstract}

Key words. Wireless medical sensor network, Gossiping, Key management.

\section{Introduction}

Following the improvement of wireless technologies and embedded systems, the potential of wireless sensor networks (WSNs) for various applications has been drawing a great deal of attention from the academia and the industry. For WSNs, one of the promising applications is healthcare. A wireless medical sensor network (WMSN, sometimes also called body sensor network) [19], which can be developed from a WSN, is a developing technology for long term monitoring of biological events or any abnormal condition of patients for realizing Ambient Assisted Living (AAL) [1]. In general, a WMSN is a moderate-scale wireless network of low-cost sensors. The purpose of WMSN is to monitor the user's physiological 
parameters and the related information in environment, e.g., ECG, EMG, EEG, $\mathrm{SpO}_{2}$ and blood pressure. The collected data will be sent to doctors or nurses for daily diagnosis. A typical scenario of WMSN is illustrated in Figure 1.

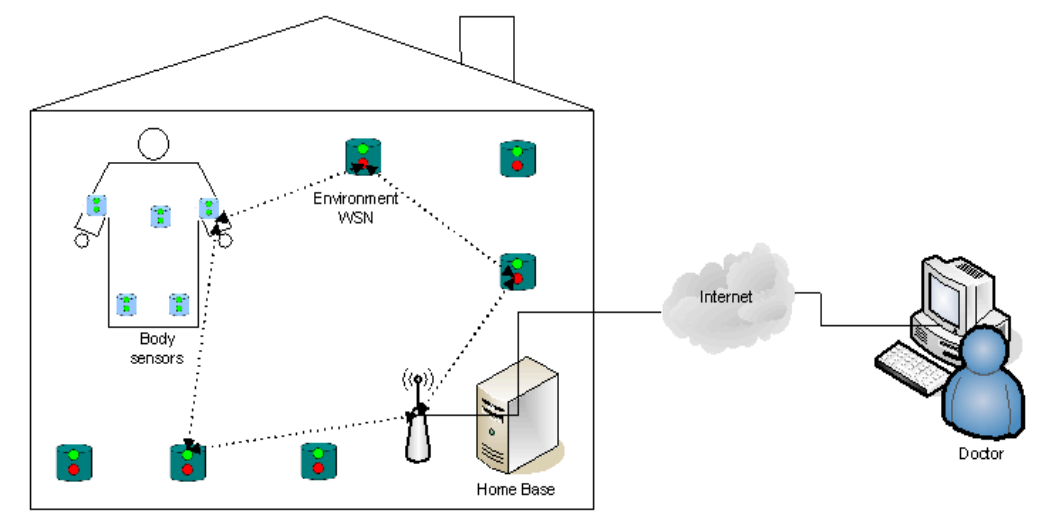

Fig. 1. A Scenario of Wireless Medical Sensor Network.

In practice, sensors used in WMSNs also have limited computational abilities and small memories, typically with a low-end CPU and RAM in KBytes level. These factors are important not only in the implantable but also in the external sensor settings because they determine how "hidden" and "pervasive" the sensors are. A gossip protocol is a style of computer-to-computer communication protocol inspired by the form of gossip seen in social networks. Since gossip-based network protocol is proven to be energy-efficient, it would be a lowcost candidate for realizing a WMSN via gossiping [10]. Recently, the ALwEN project [2] built a gossip-based wireless sensor network with 1000 nodes. The estimated lifetime of the network can be 1-2 years, which is a promising property in practice.

Although gossip-based WSN is energy-efficient, designing a appropriate key management scheme for WMSN is a challenging task. In the gossip mode, each node will send out messages to 1-hop neighbor nodes with a well-chosen probability. Thus the security model should consider the situations that all nodes can receive the message, and the message might be dropped during multi-hops. Moreover, the security and privacy problems related to healthcare systems are critical [3]. As a recent study has demonstrated, medical devices that do not support any confidentiality and authentication function are prone to eavesdropping and attacks [11]. Basically, solving these problems requires a key management scheme, which handles the cryptographic keys in a right manner, to provide data confidentiality and authenticity. In the literature, many key management schemes have been proposed for broadcast/gossip WSNs $[8,13,16]$. However, a WMSNoriented key management must consider the following differences. Firstly, in 
WMSN applications, nodes might be added or removed frequently. For the ease of a user, the initialization or revocation of such nodes should be designed as agile as possible. Since we suppose the added/removed nodes might be tampered, the resilience of compromise becomes serious in WMSN key management. Secondly, a typical WMSN is a moderate-scale WSN, so probabilistic key sharing schemes that are designed for large-scale WSNs are not suitable $[6,7,12]$. For practical applications, a good WMSN key management scheme must consider the above differences carefully, whilst balancing the applicability and the security.

Recently, Law et al. propose a novel WMSN key management scheme, which is called KALwEN [14]. But KALwEN relies on a smart Faraday cage and unicast communication channels, which might be impractical in some cases. In this work, our main contribution are two new key management schemes, namely the KALwEN+ schemes, which are secure against active and aggressive adversaries respectively. Compared to KALwEN, KALwEN+ does not require a Faraday cage, and the communication can be fully broadcast for satisfying gossip-based networks. Based on Shamir's secret sharing technique, KALwEN+ schemes support an efficient way to add/remove nodes. Using formal analysis, we prove that the KALwEN+ schemes are secure in our formalized security model. Based on their theoretical performances, the KALwEN+ schemes not only fit ALwEN, but also can be tailored to other scalable wireless sensor networks based on gossiping.

The rest of this paper is organized as follows. In Section 2, we first describe the system environment, then define the security model for KALwEN+. In Section 3, we describe the KALwEN+ scheme secure against active adversaries and prove its security in our security model. In Section 4, we describe the KALwEN+ scheme secure against aggressive adversaries and prove its security in our security model. In Section 5, we present the performance analysis for KALwEN+ schemes. In Section 6, we conclude the paper.

\section{Key Distribution Schemes for Gossip-based WMSN}

In this section we first describe the system environment, then formulate the security properties of key distribution schemes which are specifically tailored to gossip-based WMSN. The security formulations follow that of Bellare and Rogaway [4].

\subsection{Environment of Gossip-based WMSN}

Due to the special setting of gossip-based WMSN as shown in Figure 2, at the beginning of the key distribution, a node denoted as the sink node is connected to 
trusted device Dev (e.g., a home-based computer) and key distribution messages will be broadcast by the sink node as an initiator. Then, the sink node and other nodes will engage in a key management scheme. The resultant session keys will be used to protect the data collection and the gossip communications.

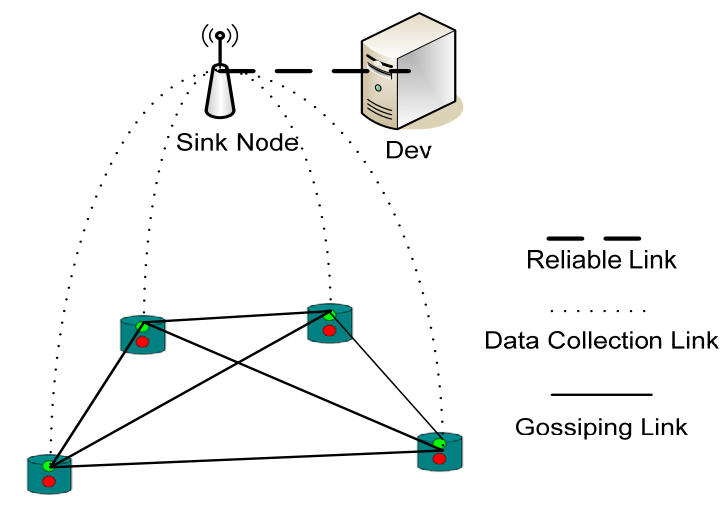

Fig. 2. Environment of Gossip-based WMSN.

\subsection{Description of Key Distribution Schemes}

We consider an environment which can consist of maximal $N$ sensor nodes, say node $_{i}(1 \leq i \leq N)$, and a trusted device Dev, such as a PC or a programmer or any other trusted infrastructure, which serves as a fully trusted third party (TTP). All nodes are honest and follow the pre-configured instructions, unless they are compromised by an adversary. In addition, we note that the trusted device Dev typically does not have the ability to connect to any node through wireless communication. To facilitate the establishment of our security model, we assume that a key distribution scheme for gossip-based WMSN consists of the following three phases.

1. System setup. In this phase, the trusted device Dev generates the long-term credentials. In the symmetric-key setting, a global key $k_{G}$ is generated, while in the public-key setting a public/private key pair $\left(P K_{G}, S K_{G}\right)$ is generated. In addition, the trusted device Dev generates some public system parameters params.

2. Node setup. In this phase, every node node $e_{i}$ is initialized by the trusted device Dev. In the symmetric-key setting, the global key $k_{G}$ is stored in the node. In the public-key setting, the trusted device Dev generates a public/private 


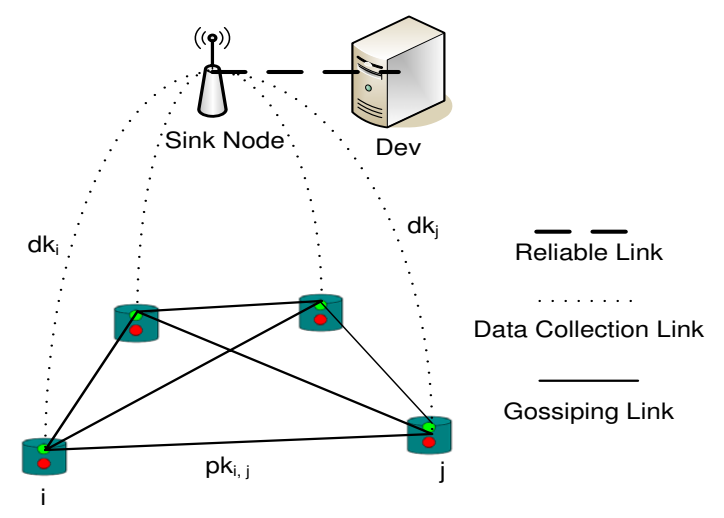

Fig. 3. Key Distribution of WMSN.

key pair $\left(P K_{i}, S K_{i}\right)$ and stores $\left(P K_{G}, C_{e r t}, P K_{i}, S K_{i}\right.$, params $)$ in node ${ }_{i}$, where Cert $_{i}$ is a certificate of $P K_{i}$ generated with $P K_{G}$.

Note that the above two steps can be executed outside the key distribution scheme. The manufacturer can generate the certificates and the global key, and then distribute them to the trusted device and the nodes beforehand.

3. Key distribution. In this phase, the following two types of session keys will be distributed to a group of nodes, say node $\left(1 \leq i \leq N^{\prime}\right)$ and $N^{\prime} \leq N$.

- The first type is data collection keys used for data collection. For node node $_{i}$, the data collection key is denoted as $d k_{i}$. This key is used for endto-end communication between node ${ }_{i}$ and the data collection gateway (namely, the sink node).

- The second type is pairwise keys used for nodes to securely communicate with each other. For a pair of nodes node $_{i}$ and node $_{j}$, the the pairwise key is denoted as $p k_{i, j}$.

In addition, we assume that the trusted device Dev keeps a counter ctr to count all the key distribution sessions. Identified by the counter $c t r$, we denote an invocation of the key distribution protocol as a session.

\subsection{Security Properties and their Formulations}

In our security model, we only consider attacks from adversaries, whose main focus is to obtain information about the session keys, including cluster keys and pairwise keys, in a certain session. We make the following assumptions:

1. No adversary is present in the system setup and node setup phases, so that no information about the long-term credentials will be leaked in both phases. 
2. An adversary may mount a denial of service (DoS) attack against the key distribution process. How to make a key distribution scheme secure in this case is beyond the scope of our model.

With respect to the secrecy of the data collection keys and pairwise keys, we consider the following types of adversaries.

- Passive Adversary $\left(\mathcal{A}^{-}\right)$. This type of adversary can only passively eavesdrop on the wireless communications in the network.

- Active Adversary $(\mathcal{A})$. This type of adversary can not only eavesdrop on, but also manipulate the wireless communications in the network. The possible manipulation of communication includes delaying, deleting, inserting, and replacing messages.

It is worth noting that both types of adversaries are outsiders since we assume all nodes are honest. In addition, since active adversaries are more powerful than the passive ones, a scheme secure against the former will also be secure against the latter.

Following the work by Bellare and Rogaway [4], the security of a key distribution scheme for gossip-based WMSN is evaluated by the attack game between a challenger and an adversary, as shown in Fig. 4, where the adversary's advantage is defined to be $\left|\operatorname{Pr}\left[b=b^{\prime}\right]-\frac{1}{2}\right|$. It is worth noting that the challenger faithfully simulates all these activities of the trusted device Dev and all the nodes.

Definition 1. A key distribution scheme for gossip-based WMSN is secure against (passive and) active adversaries, if any polynomial-time adversary has only negligible advantage in the attack game defined in Fig. 4.

It is worth stressing that in the attack game defined in Fig. 4, the adversary is allowed to obtain all data collection keys and pairwise keys in all sessions except $c t r^{*}$. As a result, a secure scheme under this definition achieves knownkey security [15].

Compared with other settings, in gossip-based WMSN, it is reasonable to assume that it is very difficult for an adversary to physically capture the nodes since they will be locked indoor or worn by patients. In other words, key distribution schemes secure against passive and active adversaries provide adequate security guarantees in most application scenarios. However, in some scenarios, higher security level may be required in the presence of an aggressive adversary $\mathcal{A}^{+}$. Besides eavesdropping on and manipulating wireless communications, this 
1. Setup: the challenger generates the parameters for the trusted device Dev and publishes the public parameters.

2. Phase 1: Besides delivering messages for all sessions, the adversary is allowed to issue the following types of queries.

(a) Invoke (set, node $\left._{i}\right)$ : The trusted device Dev initiates a new session to distribute cluster keys and pairwise keys to the nodes in the set set which is a subset of $\left\{\right.$ node $\left._{j} \mid 1 \leq j \leq N\right\}$. The node node $_{i}$ belongs to the set set and acts as the sink node.

(b) Corrupt $_{\mathrm{k}}\left(\mathrm{ctr}\right.$, node $\left._{j}\right)$ : If the session identified by $c t r$ has successfully ended and node $_{j}$ has been involved in the session, the challenger sends the data collection key and pairwise keys of node $j$ to the adversary. Otherwise, the challenger returns nothing.

At some point, the adversary chooses a counter value $c t r^{*}$ and a user index $j$, such that, in the session identified by $c t r^{*}$, node $e_{j}$ has successfully ended with $d k_{j}^{*}, p k_{j, t}^{*}$ for all $t$ such that node $t$ is also involved in the session. This is subject to the restriction that there has been no Corrupt $\left(c t r^{*}\right.$, node $\left._{t}\right)$ query for any $t$.

3. Challenge: Select $b \in_{R}\{0,1\}$. If $b=0$, send $d k_{j}^{*}, p k_{j, t}^{*}$ for all $t$ such that nodet is also involved in the session, otherwise send a replacement to the adversary, where the keys are replaced by a set of random values.

4. Phase 2: The adversary is allowed to issue the same types of queries as in Phase 1 , and is subject to the same restriction. At some point, the adversary terminates by outputting a guess bit $b^{\prime}$.

Fig. 4. The Attack Game

type of adversary is also capable of physically compromising some wireless nodes in the network even before the key management.

The security against an aggressive adversary is evaluated by the attack game between a challenger and an adversary, as shown in Fig. 5, where the adversary's advantage is defined to be $\left|\operatorname{Pr}\left[b=b^{\prime}\right]-\frac{1}{2}\right|$.

Definition 2. A key distribution scheme for WMSN is secure against an aggressive adversary, if any polynomial-time adversary has only negligible advantage in the attack game defined in Fig. 5.

It is worth stressing that in the attack game defined in Fig. 5, the adversary is allowed to obtain all data collection keys and pairwise keys in all sessions except $c t r^{*}$, and it is also allowed to obtain all long-term private keys of all nodes in Phase 2. As a result, a secure scheme under this definition achieves known-key security and perfect forward security [15]. 
1. Setup: the challenger generates the parameters for the trusted device Dev and publishes the public parameters.

2. Phase 1: Besides delivering messages for all sessions, the adversary is allowed to issue the following types of queries.

(a) Invoke (set, node $\left._{i}\right)$ : The trusted device Dev initiates a new session to distribute cluster keys and pairwise keys to the nodes in the set set which is a subset of $\left\{\right.$ node $\left._{j} \mid 1 \leq j \leq N\right\}$. The node node $_{i}$ belongs to the set set and acts as the sink node.

(b) Corrupt $_{\mathrm{k}}\left(\mathrm{ctr}\right.$, node $\left._{j}\right)$ : If the session identified by $c t r$ has successfully ended and node $_{j}$ has been involved in the session, the challenger sends the data collection key and pairwise keys of node $_{j}$ to the adversary. Otherwise, the challenger returns nothing.

(c) Corrupt $($ index $)$ : The challenger returns the long-term public/private keys of node $_{\text {index }}$ to the adversary.

At some point, the adversary chooses a counter value $c t r^{*}$ and a user index $j$, such that, in the session identified by $c t r^{*}$, node $_{j}$ has successfully ended with $d k_{j}^{*}, p k_{j, t}^{*}$ for all $t$ which satisfies that node $e_{t}$ is also involved in the session. This is subjected to the following restrictions.

(a) Suppose the node node $_{i}$ is the sink node in the session identified by $c t r^{*}$. There has been no $\operatorname{Corrupt}_{\mathrm{l}}(i)$ and $\operatorname{Corrupt}_{\mathrm{k}}\left(c^{*}{ }^{*}\right.$, node $\left._{i}\right)$ queries. The requirement also applies to node $_{j}$. Note that the adversary may choose $j=i$ in the challenge.

(b) Suppose $s^{*}$ is the set of nodes in the session identified by $c t r^{*}$ satisfying that if node $_{j} \in$ set $^{*}$ then there has been no $\operatorname{Corrupt}_{\mathrm{k}}\left(\mathrm{ctr}^{*}\right.$, node $\left._{j}\right)$ query and no $\operatorname{Corrupt}_{\mathrm{I}}(j)$ query. The size of $\operatorname{set}^{*}$ is at least 2 .

(c) In the session identified by $c t r^{*}$, at most $t-1$ nodes have been issued a Corrupt $t_{\mathrm{k}}$ query.

3. Challenge: Select $b \in_{R}\{0,1\}$. If $b=0$, send $d k_{j}^{*}, p k_{j, t}^{*}$ for all $t$ which satisfies that node $_{t}$ is also involved in the session and there has been no $\operatorname{Corrupt}_{\mathrm{k}}\left(\operatorname{ctr}^{*}\right.$, node $\left._{t}\right)$ query and and no Corrupt $(t)$ query, otherwise send a replacement to the adversary, where the keys are replaced by a set of random values.

4. Phase 2: The adversary is allowed to issue the same types of queries as in Phase 1 , with the following restriction.

(a) There has been no Corrupt $\mathrm{k}_{\mathrm{k}}\left(c t r^{*}\right.$, node $\left._{h}\right)$ query for any $h$ satisfying that there has been no Corrupt $_{\mathrm{k}}\left(\right.$ ctr $^{*}$, node $\left._{h}\right)$ query in Phase 1.

At some point, the adversary terminates by outputting a guess bit $b^{\prime}$.

Fig. 5. The Enhanced Attack Game

\section{Scheme Secure against Active Adversaries}

In this section, we propose a key distribution scheme which is secure against active adversaries. In this scheme we use symmetric key cryptographic primitives, 
including message authentication code (MAC) algorithms [15] and symmetric key encryption schemes. We make use of Shamir's secret sharing scheme [17] to deal with the issues such as adding nodes and key recovery in emergency situations.

\subsection{Preliminaries}

A MAC algorithm is a family of functions $\left\{\mathrm{MAC}_{k}\right\}$, parameterised by a secret key $k$, with the following properties:

1. Ease of computation: for a known function $\mathrm{MAC}_{k}$, given a value $k$ and an input $x, \mathrm{MAC}_{k}(x)$ is easy to compute. This result is called the MAC-value or MAC.

2. Compression: $\mathrm{MAC}_{k}$ maps an input $x$ of arbitrary finite bit-length to an output $\mathrm{MAC}_{k}(x)$ of fixed bit-length.

Definition 3. A MAC algorithm is said to be secure against existential forgery if, for any fixed key $k$ (not known to the attacker), and given any number of $M A C$ queries $\operatorname{MAC}_{k}(x)$, where the values of $x$ may be chosen by the adversary after observing the results of previous queries, a adversary can only succeed with a negligible probability in finding a pair $\left(x^{*}, \mathrm{MAC}_{k}\left(x^{*}\right)\right.$ ) where $x^{*}$ (which could be chosen by the attacker) was not in the set of MAC queries.

Shamir's secret sharing scheme [17] is based on the polynomial interpolation: given $k$ points $\left(x_{1}, y_{1}\right),\left(x_{2}, y_{2}\right), \cdots,\left(x_{k}, y_{k}\right)$, where all elements are from a finite field $\mathbb{F}$ and $x_{i}(1 \leq i \leq k)$ are distinct, there is one and only one polynomial $f(x)$ of degree $k-1$ such that $f(x)=y_{i}$ for all $i$ s. To hide a secret $d$, first pick a random $k-1$ degree polynomial $f(x)=d+a_{1} x+\cdots+a_{k-1} x^{k-1}$ and sets $d_{j}=f(j)$ for $1 \leq j \leq n$ where $n \geq k$. It is straightforward to verify that, given any subset of $k$ tuples of the set $\left\{\left(i, d_{i}\right) \mid 1 \leq i \leq n\right\}$, we can find the coefficients of $f(x)$ by interpolation and then obtain $d=f(0)$. Given just $k-1$ of these values, $d$ is indistinguishable from a random element from $\mathbb{F}$.

Let $\mathrm{F}: K \times D \rightarrow R$ be a function family, where $K=\{0,1\}^{x}, D=\{0,1\}^{y}, R=$ $\{0,1\}^{z}$ for some integers $x, y, z$. $\mathrm{F}$ is said to be a pseudorandom function family if, given the input-output behaviors, an adversary can only distinguish $\mathrm{F}(k, \cdot)$ from Ran with a negligible probability, where $k$ is randomly chosen from $\{0,1\}^{x}$ and $\operatorname{Ran}: D \rightarrow R$ is a random function [9]. 


\subsection{Description of the Scheme}

In the system setup phase, the trusted device Dev selects a symmetric encryption algorithm (ENC, DEC), an MAC algorithm MAC, and a symmetric key $k_{G}=$ $\left(k_{1}, k_{2}\right)$. It also choose a finite field $\mathbb{F}$ for Shamir's secret sharing.

In the node setup phase, $\left(k_{G}, \mathbb{F}\right)$ is stored in the node. For simplicity, we assume all nodes have been programmed to perform all the operations in the key distribution scheme. The key distribution scheme is as follows.

1. A node node $_{i}$, which is connected to the trusted device Dev, becomes a sink node, broadcasts a bootstrap message to the network. The bootstrap message is defined as follows

$$
\text { node }_{i}+\operatorname{Dev} \rightarrow *: c t r, \mathrm{ENC}_{k_{1}}\left(k_{s}\right), \mathrm{MAC}_{k_{2}}\left(1\|c t r\| \operatorname{ENC}_{k_{1}}\left(k_{s}\right)\right),
$$

where $k_{s}$ is a randomly-chosen ephemeral key for MAC.

2. After receiving the message, if the value of ctr is smaller than the local counter value, node $_{j}$ terminates by broadcasting a failure message. Otherwise, it sets the local counter value to be $c t r$, decrypts $\mathrm{ENC}_{k_{1}}\left(k_{s}\right)$, and checks

$$
\operatorname{MAC}_{k_{2}}\left(1\|c t r\| \operatorname{ENC}_{k_{1}}\left(k_{s}\right)\right) \text {. }
$$

If the MAC code is correct, it sends $\left(n_{j}, \mathrm{MAC}_{\mathrm{H}\left(1 \| k_{s}\right)}\left(2\|c t r\| I D_{j} \| n_{j}\right)\right.$ to the sink node, where $n_{j}$ is a nonce.

$$
\text { node }_{j} \rightarrow \text { node }_{i}: n_{j}, \mathrm{MAC}_{\mathrm{H}\left(1 \| k_{s}\right)}\left(2\|c t r\| I D_{j} \| n_{j}\right) .
$$

3. After receiving the message from node ${ }_{j}$, the sink node first checks the MAC code $\mathrm{MAC}_{\mathrm{H}\left(1|| k_{s}\right)}\left(2|| c t r\left\|I D_{j}\right\| n_{j}\right)$. If the check fails, it terminates by broadcasting a failure message. Otherwise, it continues. At a certain point, the sink node learns that session keys need to be distributed to a group of nodes, say node $_{j}\left(1 \leq j \leq N^{\prime}\right)$ and $N^{\prime} \leq N$. The sink node computes an ephemeral key pool $\Gamma=\left\{e k_{1}, e k_{2}, \cdots, e k_{N^{\prime}}, e k_{1}^{\prime}, e k_{2}^{\prime}, \cdots, e k_{N^{\prime}}^{\prime}\right\}$, where $1 \leq j \leq N^{\prime}, j \neq i$

(a) Using Shamir's $(t, N)$-threshold secret sharing technique, generate $N$ shares $\left\{\left(j, s h_{j}\right) \mid 1 \leq i \leq N\right\}$ to hide a secret $r \in_{R} \mathbb{F}$.

(b) Send the following message to the node node $_{j}$

$$
\begin{aligned}
\text { node }_{i} \rightarrow & \operatorname{node}_{j}: \operatorname{ENC}_{\mathrm{H}\left(2\left\|I D_{j}\right\| k_{s}\right)}\left(j\left\|s h_{j}\right\| s k_{j} \| T_{j}\right), \\
& \operatorname{MAC}_{k_{2}}\left(I D_{j}\left\|n_{j}\right\| c t r \| \operatorname{ENC}_{\mathrm{H}\left(2|| I D_{j} \| k_{s}\right)}\left(j\left\|s h_{j}\right\| s k_{j} \| T_{j}\right)\right),
\end{aligned}
$$

where $s k_{j}=\mathrm{H}\left(3\|c t r\| I D_{j} \| r\right)$ and $T_{j}$ is a concatenation of $p k_{t, j}$ for all $t$ such that $e k_{t} \in \Gamma$ and $t \neq j . p k_{t, j}$ is set to be $\mathrm{H}\left(4\|c t r\| I D_{t}\left\|I D_{j}\right\| r\right)$ if $t<j$, and $\mathrm{H}\left(4\|c t r\| I D_{j}\left\|I D_{t}\right\| r\right)$ otherwise. Consequently, $p k_{t, j}=p k_{j, t}$ holds. 
(c) The sink nodes stores $r$ and the shares $\left\{\left(j, s h_{j}\right) \mid N^{\prime}+1 \leq i \leq N\right\}$ at the trusted device Dev.

4. After receiving the message, node ${ }_{j}$ first checks the MAC code. If the check fails, it terminates by broadcasting a failure message. Otherwise, it decrypts $\mathrm{ENC}_{\mathrm{H}\left(2|| I D_{j} \| k_{s}\right)}\left(j\left\|s h_{j}\right\| s k_{j} \| T_{j}\right)$ to obtain the data collection key $s k_{j}$, pairwise keys $T_{j}$, and the share $\left(j, s h_{j}\right)$. It also updates $c t r$ to be $c t r+1$.

Lemma 1. The proposed scheme is secure under Definition 1 given that the $M A C$ algorithm is secure against existential forgery, the encryption algorithm is a pseudorandom function, and $\mathrm{H}$ is a random oracle.

Proof sketch. Suppose that an adversary has the advantage $\epsilon$ the attack game shown in Fig. 4. We first have the following observation, which implies the integrity of messages received by all nodes (the adversary is not able to manipulate the messages without being detected by some users).

Observation. During the attack game, in the session identified by ctr* (and in any other sessions), node ${ }_{j}$, for any $j$ such that node ${ }_{j}$ is involved in the session, is supposed to receive the following values:

$$
\begin{gathered}
c t r^{*}, \operatorname{ENC}_{k_{1}}\left(k_{s}\right), \operatorname{MAC}_{k_{2}}\left(1\left\|c t r^{*}\right\| \operatorname{ENC}_{k_{1}}\left(k_{s}\right)\right), \\
\operatorname{ENC}_{\mathrm{H}\left(2|| k_{s}\right)}\left(j\left\|s h_{j}\right\| s k_{j} \| T_{j}\right), \\
\operatorname{MAC}_{k_{2}}\left(I D_{j}\left\|n_{j}\right\| c t r^{*}|| \mathrm{ENC}_{\mathrm{H}\left(2 \| k_{s}\right)}\left(j\left\|s h_{j}\right\| s k_{j} \| T_{j}\right)\right),
\end{gathered}
$$

If node ${ }_{j}$ accepts the values, the probability that these values are not generated (or, simulated) by the challenger is negligible. Intuitively, the reason is that, in the proposed scheme, only sink nodes will generate messages in these format, and based on the existential forgeability of the $M A C$ algorithm an adversary can only forge such messages with a negligible probability. The proof is straightforward so that we skip it here.

The rest of the security proof is done through a sequence of games [18].

$G_{0} e_{0}$ : In this game, the challenger faithfully simulates the protocol execution and answers the oracle queries from $\mathcal{A}$. Let $\delta_{0}=\operatorname{Pr}\left[b^{\prime}=b\right]$, as we assumed at the beginning, $\left|\delta_{0}-\frac{1}{2}\right|=\epsilon$.

$\mathrm{Game}_{1}$ : The challenger performs faithfully as in $\mathrm{Game}_{0}$, except that the challenger stops if the values described in the above observation are not generated by the challenger (referred to as the event $E n t_{1}$ ). Let $\delta_{1}=\operatorname{Pr}\left[b^{\prime}=b\right]$ at the end of this game. From the Difference Lemma in [18], we have $\left|\delta_{1}-\delta_{0}\right| \leq \operatorname{Pr}\left[E n t_{1}\right]$ which is negligible. 
$G_{2} e_{2}$ : The challenger performs faithfully as in $G_{a m e}$, except that, in the session identified by $c t r *$, in step 3 of the scheme the messages sent to node $_{j}$, for any $j$ such that node $e_{j}$ is involved in the session, are replaced with the following, where $\operatorname{Ran}_{j}$ is random function.

$$
\begin{gathered}
\operatorname{Ran}_{j}\left(j\left\|s h_{j}\right\| s k_{j} \| T_{j}\right), \\
\operatorname{MAC}_{k_{2}}\left(I D_{j}\left\|n_{j}\right\| c t r \| \operatorname{Ran}_{j}\left(j\left\|s h_{j}\right\| s k_{j} \| T_{j}\right)\right),
\end{gathered}
$$

Since $\mathrm{H}$ is a random oracle and the encryption algorithm is a pseudorandom function, $\mathrm{Game}_{2}$ is identical to Game $\mathrm{H}_{1}$ unless the adversary queries $\mathrm{H}$ with $*\left\|k_{s}\right\| *$ (referred to as the event $E n t_{2}$ ), where $*$ can be any string. Furthermore, since the encryption algorithm is a pseudorandom function, $\operatorname{Pr}\left[E n t_{2}\right]$ is negligible. Let $\delta_{2}=\operatorname{Pr}\left[b^{\prime}=b\right]$ at the end of this game. From the Difference Lemma in [18], we have $\left|\delta_{2}-\delta_{1}\right| \leq \operatorname{Pr}\left[E n t_{2}\right]$ which is negligible.

In Game 2 , since the encryption of the session keys and shares is provided by random functions, the probability $\delta_{2}=\frac{1}{2}$. As a result, we have

$$
\begin{aligned}
\epsilon & =\left|\delta_{0}-\frac{1}{2}\right| \\
& \leq\left|\delta_{1}-\delta_{0}\right|+\left|\delta_{2}-\delta_{1}\right|+\left|\delta_{2}-\frac{1}{2}\right| \\
& \leq \operatorname{Pr}\left[E_{1} t_{1}\right]+\operatorname{Pr}\left[E_{2} t_{2}\right]
\end{aligned}
$$

Since $\operatorname{Pr}\left[E n t_{1}\right]$ and $\operatorname{Pr}\left[E n t_{2}\right]$ are negligible, the lemma now follows.

\subsection{Further Remarks}

If a key distribution execution has been carried out for node n $_{j}\left(1 \leq j \leq N^{\prime}\right)$, later on $\operatorname{node}_{v}$ for any $N^{\prime}+1 \geq v \geq N$ may need to join the communications. With respect to the key distribution scheme, there are two possibilities to add a new node into a group. Note the fact that node ${ }_{v}$ should have been initialized and share the key $K_{G}$ with the trusted device Dev.

In the first case, if Dev is available, then it can just generate the corresponding data collection key and pairwise keys for node $e_{v}$ based on the secret value $r$ and sends these keys and a share $\left(v, s h_{v}\right)$ to node $e_{v}$ through a secure channel provided by the shared long-term key $K_{G}$.

In the second case, if Dev is unavailable, then the secret $r$ can be recovered by node $\operatorname{nos}_{j}\left(1 \leq j \leq N^{\prime}\right)$ using their shares $\left(j, s h_{j}\right)\left(1 \leq j \leq N^{\prime}\right)$. Then the corresponding data collection key and pairwise keys for node can $_{v}$ be generated and transmitted to node $v$ in the same way as the above case. 


\section{Scheme Secure against Aggressive Adversaries}

In this section, we propose a key distribution scheme which is secure against aggressive adversaries. Compared with the previous scheme, we use public key cryptographic techniques, including digital signature schemes and Diffie-Hellman key exchange, in order to deter the effect of compromised nodes by aggressive adversaries. Nonetheless, both key distribution schemes make use of the secret sharing technique, therefore, the remarks in Section 3.3 apply to this scheme and we skip it here ${ }^{4}$.

\subsection{Preliminaries}

Digital signature schemes provide a means by which an entity can bind its identity (or public key) to a piece of information (usually referred to as a message). A digital signature scheme is made up of the following algorithms [15]:

1. KeyGen: which takes a security parameter $\ell$ as input, and outputs a public (verification) key $p k$ and a private (signing) key $s k$.

2. Sign: which takes as input a message $m$ and a private key $s k$ and produces a signature $\sigma$ for the message $m$.

3. Verify: which takes as input a message $m$, a public key $p k$ and a signature $\sigma$, and outputs either accept (denoted by 1 ) or reject (denoted by 0 ).

The existential unforgeability of a digital signature scheme is defined as follows:

Definition 4. A digital signature scheme is existentially unforgeable under an adaptive chosen message attack if the probability of success of any polynomially bounded attacker in the following game is negligible. The attack game is carried out between an attacker $\mathcal{A}$ and the hypothetical challenger $\mathcal{C}$.

1. Initialisation: $\mathcal{C}$ runs $\operatorname{KeyGen}(\ell)$ to generate a public key $p k$ and a private key sk.

2. Challenge: The attacker runs $\mathcal{A}$ on the input pk and terminates by outputting a pair $m^{*}, \sigma^{*}$. During its execution, $\mathcal{A}$ can query the Sign oracle with any input $m\left(m \neq m^{*}\right)$.

The attacker wins the game if $\operatorname{Verify}\left(m^{*}, p k, \sigma^{*}\right)=1$, and, the attacker's advantage is defined to be $\operatorname{Pr}\left[\operatorname{Verify}\left(m^{*}, p k, \sigma^{*}\right)=1\right]$.

\footnotetext{
${ }^{4}$ The only difference is that a secure channel between Dev and a new node can be provided using a symmetric key resulted from a standard Diffie-hellman key exchange.
} 
Given a group $\mathbb{G}$ of order $p$, the computational Diffie-Hellman assumption holds if, given $g^{x}$ and $g^{y}$ where $x, y$ are randomly chosen from $\mathbb{Z}_{p}$, an adversary can compute $g^{x y}$ only with a negligible probability.

\subsection{Description of the Proposed Scheme}

In the system setup phase, the trusted device Dev selects a digital signature algorithm (KeyGen, Sign, Verify) and a public/private key pair $\left(P K_{G}, S K_{G}\right)$. It also chooses a group $\mathbb{G}$ for Diffie-Hellman key exchange [5] and a finite field $\mathbb{F}$ for Shamir's secret sharing.

In the node setup phase, every node node $_{i}$ is initialized by the trusted device Dev: a public/private key pair $\left(P K_{i}, S K_{i}\right)$ is generated and the parameters $\left(P K_{G}, C_{e r t}, P K_{i}, S K_{i}, \mathbb{G}, \mathbb{F}\right)$ are stored in the node, where $C_{e r t}$ is a signature of $P K_{i} \| I D_{i}$ signed with $S K_{G}$. For simplicity, we assume all nodes have been programmed to perform all the operations in the key distribution scheme. The key distribution scheme is as follows.

1. A node node $_{i}$, which is connected to the trusted device Dev, becomes a sink node, broadcasts a bootstrap message to the network. The bootstrap message is defined as follows.

$$
\operatorname{node}_{i}+\operatorname{Dev} \rightarrow *: c t r, g^{r_{i}}, \operatorname{Sign}_{S K_{G}}\left(c t r \| g^{r_{i}}\right) .
$$

2. After receiving the bootstrap message, every node node $_{j}$ verifies the signature. If the signature is not valid or the value of $c t r$ is smaller than the local counter value, node $_{j}$ terminates by broadcasting a failure message. Otherwise, it sets its local counter value to be $c t r$, and sends the following message to the sink node.

$$
\text { node }_{j} \rightarrow \text { node }_{i}: g^{r_{j}}, \operatorname{Sign}_{S_{K}}\left(c t r\left\|g^{r_{i}}\right\| g^{r_{j}}\right) .
$$

The node node $_{j}$ computes two ephemeral keys $e k_{j}$ and $e k_{j}^{\prime}$, where

$$
e k_{j}=\mathrm{H}\left(1\left\|g^{r_{i} r_{j}}\right\| c t r\left\|I D_{i}\right\| I D_{j}\right), e k_{j}^{\prime}=\mathrm{H}\left(2\left\|g^{r_{i} r_{j}}\right\| c t r\left\|I D_{i}\right\| I D_{j}\right) .
$$

3. After receiving the message from node ${ }_{j}$, the sink node first checks the counter value and the signature. If the check fails, it terminates by broadcasting a failure message. Otherwise, it continues. At a certain point, the sink node learns that session keys need to be distributed to a group of nodes, say node ${ }_{j}$ $\left(1 \leq j \leq N^{\prime}\right)$ and $N^{\prime} \leq N$. The sink node computes an ephemeral key pool $\Gamma=\left\{e k_{1}, e k_{2}, \cdots, e k_{N^{\prime}}, e k_{1}^{\prime}, e k_{2}^{\prime}, \cdots, e k_{N^{\prime}}^{\prime}\right\}$, where for $1 \leq j \leq N^{\prime}, j \neq i$

$$
e k_{j}=\mathrm{H}\left(1\left\|g^{r_{i} r_{j}}\right\| c t r\left\|I D_{i}\right\| I D_{j}\right), e k_{j}^{\prime}=\mathrm{H}\left(2\left\|g^{r_{i} r_{j}}\right\| c t r\left\|I D_{i}\right\| I D_{j}\right) \text {. }
$$

The sink node then does the following. 
(a) Using Shamir's $(t, N)$-threshold secret sharing technique, generate $N$ shares $\left\{\left(j, s h_{j}\right) \mid 1 \leq j \leq N\right\}$ to hide a secret $r \in_{R} \mathbb{F}$.

(b) Send the following message to the node node $_{j}$

$$
\begin{aligned}
\text { node }_{i} \rightarrow & \text { node }_{j}: \operatorname{ENC}_{e k_{j}}\left(c t r\|j\| s h_{j}\left\|s k_{j}\right\| T_{j}\right), \\
& \operatorname{MAC}_{e k_{j}^{\prime}}\left(c t r \| \operatorname{ENC}_{e k_{j}}\left(c t r\left\|s k_{j}\right\| T_{j}\right)\right),
\end{aligned}
$$

where $s k_{j}=\mathrm{H}\left(3\|c t r\| I D_{j} \| r\right)$ and $T_{j}$ is a concatenation of $p k_{t, j}$ for all $e k_{t} \in \Gamma$ and $t \neq i$. The value $p k_{t, j}$ is set to be $\mathrm{H}\left(4\|c t r\| I D_{t}\left\|I D_{j}\right\| r\right)$ if $t<j$, and $\mathrm{H}\left(4\|c t r\| I D_{j}\left\|I D_{t}\right\| r\right)$ otherwise. Consequently, $p k_{t, j}=p k_{j, t}$ holds.

4. After receiving the message, node ${ }_{j}$ first checks the MAC code. If the check fails, it terminates by broadcasting a failure message. Otherwise, it decrypts $\mathrm{ENC}_{e k_{j}}\left(j\left\|s h_{j}\right\| s k_{j} \| T_{j}\right)$ to obtain the data collection key $s k_{j}$, pairwise keys $T_{j}$, and the share $\left(j, s h_{j}\right)$. It also update $c t r$ to be $c t r+1$.

Lemma 2. The proposed scheme is secure under Definition 2 based on the computational Diffie-Hellman (CDH) assumption, given that the digital signature scheme is existentially unforgeable, the encryption algorithm is a pseudorandom function, and $\mathrm{H}$ is a random oracle.

Proof sketch. Suppose that an adversary has the advantage $\epsilon$ the attack game shown in Fig. 5. We first have the following observation.

Observation. During the attack game, in the session identified by ctr*, node $_{j}$, for any $j$ such that node ${ }_{j}$ is involved in the session, is supposed to receive the following value:

$$
\begin{gathered}
c t r^{*}, g^{r_{i}}, \operatorname{Sign}_{S K_{G}}\left(c t r^{*} \| g^{r_{i}}\right), \\
\operatorname{ENC}_{e k_{j}}\left(c t r^{*}\|j\| s h_{j}\left\|s k_{j}\right\| T_{j}\right), \operatorname{MAC}_{e k_{j}^{\prime}}\left(c t r^{*} \| \mathrm{ENC}_{e k_{j}}\left(c t r^{*}\left\|s k_{j}\right\| T_{j}\right)\right) .
\end{gathered}
$$

Based on the existential unforgeability of the signature scheme, the probability that the first message is not generated (or, simulated) by the challenger is negligible. Based on the CDH assumption and the existential unforgeability of the MAC algorithm, the probability that an adversary can forge the second message is negligible given that $\mathrm{H}$ is a random oracle. Therefore, these values are generated by the challenger, and the proof is straightforward so that we skip it here.

The rest of the security proof is done through a sequence of games [18].

Game $_{0}$ : In this game, the challenger faithfully simulates the protocol execution and answers the oracle queries from $\mathcal{A}$. Let $\delta_{0}=\operatorname{Pr}\left[b^{\prime}=b\right]$, as we assumed at the beginning, $\left|\delta_{0}-\frac{1}{2}\right|=\epsilon$. 
$G_{1} e_{1}$ : The challenger performs faithfully as in Game ${ }_{0}$, except that the challenger stops if the values described in the above observation are not generated by the challenger (referred to as the event $E n t_{1}$ ). Let $\delta_{1}=\operatorname{Pr}\left[b^{\prime}=b\right]$ at the end of this game. From the Difference Lemma in [18], we have $\left|\delta_{1}-\delta_{0}\right| \leq \operatorname{Pr}\left[E n t_{1}\right]$ which is negligible.

Game 2 : The challenger performs faithfully as in Game ${ }_{1}$, except that, in the session identified by $c t r^{*}$, in step 3 of the scheme the messages sent to node $_{j}$, for any $j$ such that node $_{j}$ is involved in the session and node ${ }_{j}$ has not been issued any Corrupt $t_{1}$ query, are replaced with the following, where $\operatorname{Ran}_{j}$ is a random function.

$$
\begin{gathered}
\operatorname{Ran}_{j}\left(j\left\|s h_{j}\right\| s k_{j} \| T_{j}\right), \\
\operatorname{MAC}_{e k_{j}^{\prime}}\left(I D_{j}\left\|c t r^{*}\right\| \operatorname{Ran}_{j}\left(j\left\|s h_{j}\right\| s k_{j} \| T_{j}\right)\right),
\end{gathered}
$$

Since $\mathrm{H}$ is a random oracle and the encryption algorithm is a pseudorandom function, Game $e_{2}$ is identical to Game ${ }_{1}$ unless the event Ent $t_{2}$ occurs: the adversary has queried $\mathrm{H}$ with $*\|r\| *$ or $*\left\|g^{r_{i} r_{j}}\right\| *$ for any $j$ such that node ${ }_{j}$ has not been issued any Corrupt $\mathrm{I}_{\mathrm{I}}$ query. Based on the $\mathrm{CDH}$ assumption and the security of the Shamir secret sharing scheme, $\operatorname{Pr}\left[E n t_{2}\right]$ is negligible. Let $\delta_{2}=\operatorname{Pr}\left[b^{\prime}=b\right]$ at the end of this game. From the Difference Lemma in [18], we have $\left|\delta_{2}-\delta_{1}\right| \leq \operatorname{Pr}\left[E n t_{2}\right]$ which is negligible.

In $\mathrm{Game}_{2}$, since the encryption is provided by random functions, the probability $\delta_{2}=\frac{1}{2}$. As a result, we have

$$
\begin{aligned}
\epsilon & =\left|\delta_{0}-\frac{1}{2}\right| \\
& \leq\left|\delta_{1}-\delta_{0}\right|+\left|\delta_{2}-\delta_{1}\right|+\left|\delta_{2}-\frac{1}{2}\right| \\
& \leq \operatorname{Pr}\left[E^{2} t_{1}\right]+\operatorname{Pr}\left[E_{2} t_{2}\right]
\end{aligned}
$$

Since $\operatorname{Pr}\left[E n t_{1}\right]$ and $\operatorname{Pr}\left[E n t_{2}\right]$ are negligible, the lemma now follows.

\section{Performance Analysis}

Based on the theoretical results, here we give a performance analysis of KALwEN+. Let $T_{e}$ be the time for a symmetric key encryption, and $T_{m}$ be the time for computing a MAC value. Let $T_{p}$ be time for one exponentiation computation. $T_{s}$ denotes the time for the $(t, N)$-threshold secret sharing algorithm which is used in KALwEN+. Let $T_{s i g}$ and $T_{v e r}$ be the time costs for generating and verifying a signature, respectively. For a gossip sensor network with $n$ nodes, the performance of KALwEN+ is estimated as follows. 
Table 1. The Performance Estimation of KALwEN+.

\begin{tabular}{|c|c|c|}
\hline KALwEN+ & Against Active Adversary & Against Aggressive Adversary \\
\hline Sink node costs & $(\mathrm{n}+1) T_{e}+(\mathrm{n}+1) T_{m}+1 T_{s}$ & $1 T_{s i g}+(\mathrm{n}+1) T_{p}+\mathrm{n} T_{e}+\mathrm{n} T_{m}+1 T_{s}$ \\
\hline Member node costs & $2 T_{m}+2 T_{e}$ & $1 T_{v e r}+1 T_{s i g}+1 T_{p}+1 T_{e}+1 T_{m}$ \\
Communication rounds & 3 -Rounds & 3 -Rounds \\
Storage costs & $\mathrm{O}(\mathrm{n})$ & $\mathrm{O}(\mathrm{n})$ \\
\hline
\end{tabular}

For the estimated performance, the potential bottleneck of the scheme will be the sink node. Especially in a large network, a typical sensor node can hardly afford the computational costs of $(t, N)$-threshold secret sharing by itself. Since the sink node can be connected to a trusted device, the computational costs would possibly be shared by the device while the scalability of network is large.

\section{Conclusion}

By simply using the Shamir's secret sharing techniques and the Diffie-Hellman algorithm, a family of novel key management schemes that named KALwEN+ has been proposed for wireless medical sensor network. The KALwEN+ schemes can be fully based on broadcast communication, and does not require special equipment like some existing schemes do. The secret sharing technique used in KALwEN+ not only supports efficient node addition/removal, but also elegantly ensures security against key-exposure. For applications with highly-constrained resources, the KALwEN+ scheme that fully based on symmetric cryptographic primitives is a reasonable choice. For future work, we will investigate the practical performance and the interoperability of KALwEN+ in a multi-user scenario.

Acknowledgement. We would like to thank Frits van der Wateren and Teun Hendriks for their helpful advice during ALwEN workshops. And also thank many anonymous reviewers for their valuable comments. Zheng Gong acknowledges the support of SenterNovem for the ALwEN project, grant PNE07007. Yee Wei Law is supported by the Australian Research Council Research Network on Intelligent Sensors, Sensor Networks and Information Processing (ISSNIP), and the ARC DP1095452.

\section{References}

1. AAL. European union. the ambient assisted living (aal) joint programme. http://www.aal-europe.eu/about-aal, January 2008.

2. ALwEN. Ambient living with embedded networks. http://www.alwen.nl, January 2010. 
3. R. Anderson. A security policy model for clinical information systems. IEEE Symposium on Security and Privacy, pages 30-43, 1996.

4. M. Bellare and P. Rogaway. Entity authentication and key distribution. In D. R. Stinson, editor, Advances in Cryptology - CRYPTO 1993, volume 773 of Lecture Notes in Computer Science, pages 110-125. Springer, 1993.

5. W. Diffie and M. Hellman. New directions in cryptography. IEEE Transactions on Information Theory, IT-22(6):644-654, 1976.

6. R. Dutta, E.-C. Chang, and S. Mukhopadhyay. Efficient self-healing key distribution with revocation for wireless sensor networks using one way key chains. In ACNS '07: Proceedings of the 5th international conference on Applied Cryptography and Network Security, pages 385-400, Berlin, Heidelberg, 2007. Springer-Verlag.

7. R. Dutta, S. Mukhopadhyay, and T. Dowling. Generalized self-healing key distribution in wireless adhoc networks with trade-offs in user's pre-arranged life cycle and collusion resistance. In Q2SWinet '09: Proceedings of the 5th ACM symposium on QoS and security for wireless and mobile networks, pages 80-87, New York, NY, USA, 2009. ACM.

8. L. Eschenauer and V. D. Gligor. A key-management scheme for distributed sensor networks. In CCS '02: Proceedings of the 9th ACM conference on Computer and communications security, pages 41-47, New York, NY, USA, 2002. ACM.

9. O. Goldreich, S. Goldwasser, and S. Micali. How to construct random functions. J. ACM, 33(4):792-807, 1986.

10. Z. J. Haas, J. Y. Halpern, and L. Li. Gossip-based ad hoc routing. IEEE/ACM Transactions on Networking (TON), 14(3):479-491, 2006.

11. D. Halperin, T. S. Heydt-Benjamin, B. Ransford, S. S. Clark, B. Defend, W. Morgan, K. Fu, T. Kohno, and W. H. Maisel. Pacemakers and implantable cardiac defibrillators: Software radio attacks and Zero-Power defenses. In 29th IEEE Symposium on Security and Privacy, pages 129-142, Oakland, California, May 2008. IEEE Computer Society.

12. F. Kausar, S. Hussain, J. H. Park, and A. Masood. Secure group communication with self-healing and rekeying in wireless sensor networks. In $M S N^{\prime} 0 \%$ : Proceedings of the 3rd international conference on Mobile ad-hoc and sensor networks, pages 737-748, Berlin, Heidelberg, 2007. Springer-Verlag.

13. A. Khalili, J. Katz, and W. A. Arbaugh. Toward secure key distribution in truly adhoc networks. Applications and the Internet Workshops, IEEE/IPSJ International Symposium on, 0:342, 2003.

14. Y. Law, G. Moniava, Z. Gong, P. Hartel, and M. Palaniswami. KALwEN: A New Practical and Interoperable Key Management Scheme for Body Sensor Networks. Security and Communication Networks, in press 2010.

15. A. J. Menezes, P. C. van Oorschot, and S. A. Vanstone. Handbook of Applied Cryptography. CRC Press, 1997.

16. L. B. Oliveira, H. C. Wong, M. Bern, R. Dahab, and A. A. F. Loureiro. Secleach a random key distribution solution for securing clustered sensor networks. Network Computing and Applications, IEEE International Symposium on, 0:145-154, 2006.

17. A. Shamir. How to share a secret. Commun. ACM, 22(11):612-613, 1979.

18. V. Shoup. Sequences of games: a tool for taming complexity in security proofs. http://shoup.net/papers/, 2006.

19. G. Z. Yang. Body Sensor Network. Springer London, 2003. 